\title{
A Method for Generating A Notification On A Mobile Device Is Provided
}

\author{
Jie Wang, Chuansheng Wu \\ Software College, University of Science and Technology Liaoning,China
}

gykwcs@163.com

Keywords: based; activity detected; activity monitoring; device

\begin{abstract}
Methods, systems and devices are provided for motion-activated display of messages on an activity monitoring device. In one embodiment, method for presenting a message on an activity monitoring device is provided, including the following method operations: downloading a plurality of messages to the device; detecting a stationary state of the device; detecting a movement of the device from the stationary state; in response to detecting the movement from the stationary state, selecting one of a plurality of messages, and displaying the selected message on the device.
\end{abstract}

\section{Introduction}

In recent years, the need for health and fitness has grown tremendously. The growth has occurred due to a better understanding of the benefits of good fitness to overall health and wellness. Unfortunately, although today's modern culture has brought about many new technologies, such as the Internet, connected devices and computers, people have become less active. Additionally, many office jobs require people to sit in front of computer screens for long periods of time, which further reduces a person's activity levels. Furthermore, much of today's entertainment options involve viewing multimedia content, computer social networking, and other types of computer involved interfacing. Although such computer activity can be very productive as well as entertaining, such activity tends to reduce a person's overall physical activity[1].

\section{Method for generating a notification on a mobile device is provided}

A method for generating a notification on a mobile device is provided including: establishing a wireless connection to an activity monitoring device; receiving activity data from the activity monitoring device via the wireless connection; processing the activity data to determine an activity metric for a user of the activity monitoring device; comparing the activity metric against a predefined threshold, the predefined threshold being mapped to a notification message; in response to determining that the activity metric reaches or exceeds the predefined threshold, scheduling the notification message for display on the mobile device at a specified date and time; wherein the method is executed by at least one processor[2,3].

A server-executed method for presenting a notification on a mobile device is provided, including: establishing communication with the mobile device; receiving activity data from the mobile device, the activity data being processed by the mobile device from logged data received from an activity monitoring device; processing the activity data to determine an activity metric for a user of the activity monitoring device; comparing the activity metric against a predefined threshold, the predefined threshold being mapped to a notification message; in response to determining that the activity metric reaches or exceeds the predefined threshold, sending the notification message to a notification service, the notification service configured to transmit the notification message to the mobile device for rendering on the mobile device, wherein rendering the notification message includes scheduling the notification message for a time window; wherein the method is executed by at least one processor[4].

\section{An activity tracking device}

FIG. 1 shows a block diagram of an activity tracking device, in accordance with one 
embodiment of the present invention. The activity tracking device is contained in a housing, which may be worn or held by a user. The housing may be in the form of a wristband, a clip on device, a wearable device, or may be held by the user either in the user's hand or in a pocket or attached to the user's body. The activity tracking device includes device components, which may be in the form of logic, storage, and glue logic, one or more processors, microelectronics, and interfacing circuitry. In one example, the components will include a processor, memory, a wireless transceiver, a user interface, biometric sensors, and environmental sensors .

The environmental sensors may be in the form of motion detecting sensors. In some embodiments, a motion sensor can be one or more of an accelerometer, or a gyroscope, or a rotary encoder, or a calorie measurement sensor, or a heat measurement sensor, or a moisture measurement sensor, or a displacement sensor, or an ultrasonic sensor, or a pedometer, or an altimeter, or a linear motion sensor, or an angular motion sensor, or a multi-axis motion sensor, or a combination thereof. The biometric sensors can be defined to measure physiological characteristics of the user that is using the activity tracking device. The user interface provides a way for communicating with the activity tracking device, in response to user interaction . The user interaction can be in the form of physical contact[5].



FIG1. shows a block diagram of an activity tracking device

In some embodiments, the user interface is configured to receive user interaction by way of proximity sensors, button presses, touch sensitive screen inputs, graphical user interface inputs, voice inputs, sound inputs, etc. The activity tracking device can communicate with a client and/or server using the wireless transceiver. The wireless transceiver will allow the activity tracking device to communicate using a wireless connection, which is enabled by wireless communication logic. The wireless communication logic can be in the form of a circuit having radio communication capabilities. The radio communication capabilities can be in the form of a Wi-Fi connection, a Bluetooth connection, a low-energy Bluetooth connection, or any other form of wireless tethering or near field communication. In still other embodiments, the activity tracking device can communicate with other computing devices using a wired connection (not shown). As mentioned, the environmental sensors can detect motion of the activity tracking device .

\section{Activity tracking device in communication with a remote device}

FIG. 2 illustrates an example of activity tracking device in communication with a remote device . Remote device is a computing device that is capable of communicating wirelessly with activity tracking device and with the Internet 160. Remote device can support installation and execution of applications. Such applications can include an activity tracking application . Activity tracking application can be downloaded from a server. The server can be a specialized server or a server that provides applications to devices, such as an application store. Once the activity tracking application is installed in the remote device, the remote device can communicate or be set to communicate with activity tracking device (Device A). The remote device can be a smartphone, 
a handheld computer, a tablet computer, a laptop computer, a desktop computer, or any other computing device capable of wirelessly interfacing with Device A and the Internet.

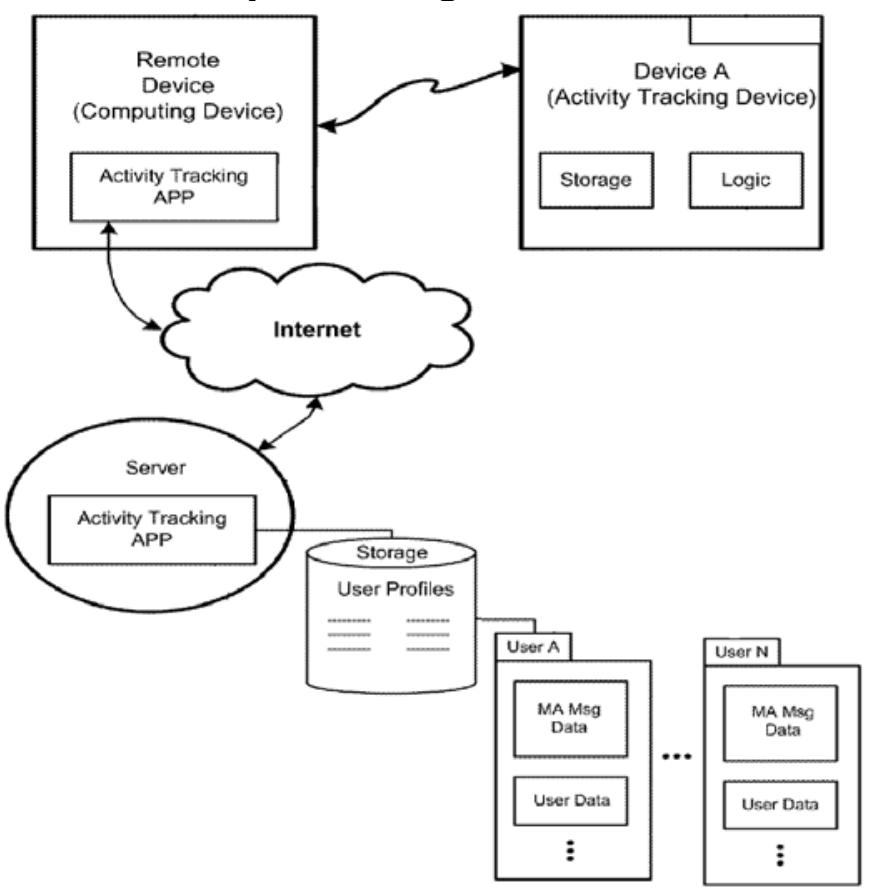

FIG. 2 illustrates an example of activity tracking device in communication with a remote device

In one embodiment, remote device communicates with activity tracking device over a Bluetooth connection. In one embodiment, the Bluetooth connection is a low energy Bluetooth connection (e.g., Bluetooth LE, BLE, or Bluetooth Smart). Low energy Bluetooth is configured for providing low power consumption relative to standard Bluetooth circuitry. Low energy Bluetooth uses, in one embodiment, a $2.4 \mathrm{GHz}$ radio frequency, which allows for dual mode devices to share a single radio antenna. In one embodiment, low energy Bluetooth connections can function at distances up to 50 meters, with over the air data rates ranging between 1-3 megabits (Mb) per second. In one embodiment, a proximity distance for communication can be defined by the particular wireless link, and is not tied to any specific standard. It should be understood that the proximity distance limitation will change in accordance with changes to existing standards and in view of future standards and/or circuitry and capabilities.

\section{Conclusion}

Although the method operations were described in a specific order, it should be understood that other housekeeping operations may be performed in between operations, or operations may be performed in an order other than that shown, or operations may be adjusted so that they occur at slightly different times, or may be distributed in a system which allows the occurrence of the processing operations at various intervals associated with the processing.

\section{References}

[1] Yue Hu,Jiejing Yu,Jiangyan Du. Determination of Mitoxantrone with an electropolymerized molecular imprinted polymer electrochemical sensor[A]. Proceedings of The 8th International Conference on Molecular Imprinting (MIP2014) Session 2[C]. 2014

[2] Guifeng Lin,Yanhong Wang,Guochen Li,Wen Bai,Hong Zhang,Shicheng Wang. Construction and Application of Molecularly Imprinted Film Sensor on Determination of Chlorpyrifos in Water[A]. Materials Science and Engineering Technology[C]. 2014

[3] Shamshiya Amerkhanova,Dana Belgibayeva,Rustam Shlyapov,Aitolkyn Uali. The Electrode for Potentiometric Determination of Chromium (III, VI) in Water Solutions[A]. Proceedings of 
2014 Conference on Physical Chemistry[C]. 2014

[4] wagiha H.Mahmoud,Eman assirey. PVC matrix membrane sensor based on prometryn for selective determination of mercury(II) ions[A]. Abstracts of the 1st International Congress on Advanced Materials[C]. 2011

[5] S.K.Shukla,Ashutosh Tiwari,Sudhish K.Shukla,Ajay $\mathrm{Kr}$ Mishra. Novel Zinc Oxide Encapsulated Chitosan/Polyvinyl Alcohol Composite for Glucose Sensing[A]. Abstracts of the 1st International Congress on Advanced Materials[C]. 2011 